\title{
How coronal alignment affects distal femoral anatomy: an MRI-based comparison of varus and valgus knees
}

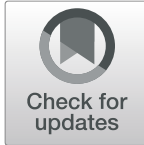

\author{
Daniel A Cohen * ${ }^{*}$, Ali C Gursel and Adrian K Low
}

\begin{abstract}
Purpose: In contemporary total knee arthroplasty (TKA), most often, the goal is to align the femoral component to the epicondylar axis (EA). The posterior condylar axis (PCA) is easier to define than the EA, and thus the relationship of PCA to the EA is then used instead to align the femoral component to the EA. However, the relationship of PCA to EA is not constant and has been reported to differ between varus and valgus knees and with increasing deformity. The aim of this large MRI-based study was to evaluate the relationship between PCA and EA with varying coronal deformity especially with increasing valgus deformity.

Methods: EA, PCA, AP (Whiteside's line) and the mechanical axis were obtained from 474 magnetic resonance imaging (MRI) scans used to create patient-specific instrumentation (PSI) for the Biomet Signature (Warsaw, NJ) system.

Results: The relationship of EA relative to the PCA showed considerable heterogeneity in both varus and valgus groups. In the valgus group, there was statistically greater external rotation $(P<0.05)$ of the EA from the PCA with a mean of $2.52^{\circ}$ (range $-1.9^{\circ}$ to $6^{\circ}$ ) compared to the varus group with a mean of $2.03^{\circ}$ (range $-3.9^{\circ}$ to $6.9^{\circ}$ ). This relationship did not significantly change with increasing severity of coronal malalignment. Externally rotating the femoral cutting guide by $3^{\circ}$ from the PCA, 11\% (42 of 382) of varus knees would lie outside of $\pm 3^{\circ}$ from EA. In valgus knees, externally rotating the femoral cutting block by $3^{\circ}$ or $5^{\circ}$ from the PCA, $6.5 \%$ (6 of 92) and 33.7\% (31 of 92) of knees, respectively, would lie outside of $\pm 3^{\circ}$ from EA.

Conclusion: The relationship of PCA to EA is heterogeneous and is not altered significantly with increasing valgus coronal deformity. External rotation beyond $3^{\circ}$ from PCA in valgus knees may lead to significant femoral component malrotation in a large proportion cases.
\end{abstract}

Keywords: Valgus knee, Lateral femoral condyle, Femoral component rotation, Posterior condylar axis, Epicondylar axis

\section{Introduction}

Successful total knee arthroplasty (TKA) relies on accurate placement of femoral and tibial components in the sagittal, coronal and axial plane to restore the mechanical axis of the limb and soft tissue tension within the knee. With respect to the axial rotation of the femoral component, various axes including the epicondylar axis (EA), the anteroposterior axis or Whiteside's line (AP),

\footnotetext{
* Correspondence: dcohen44@hotmail.com

Specialty Orthopaedics, Norwest Private Hospital, G18/11 Norbrik Drive Bella Vista, Sydney, NSW 2153, Australia (a) Sydney, NSW 2153, Australia
}

C The Author(s). 2019 Open Access This article is distributed under the terms of the Creative Commons Attribution 4.0 International License (http://creativecommons.org/licenses/by/4.0/), which permits unrestricted use, distribution, and reproduction in any medium, provided you give appropriate credit to the original author(s) and the source, provide a link to the Creative Commons license, and indicate if changes were made. The Creative Commons Public Domain Dedication waiver (http://creativecommons.org/publicdomain/zero/1.0/) applies to the data made available in this article, unless otherwise stated.

and the posterior condylar axis (PCA) assist in correct positioning [1-3].

In contemporary TKA, most often, the femoral component is aligned parallel to the EA. The EA is generally considered the axis about which flexion and extension occurs, and rotation parallel to the EA creates a balanced flexion gap relative to a perpendicular tibial cut. However, this axis is difficult to define accurately intraoperatively [4]. In contrast, the posterior femoral condyles and therefore the PCA are better defined. As such, the relationship of the PCA to the EA is used as a 
surrogate to determine the axial rotation of the femoral component.

Studies have shown that the PCA is on average $3^{\circ}$ internally rotated relative to the EA in normal knees [5]. Therefore, when conventional alignment jigs are used, the femoral cutting block is usually externally rotated from the PCA by $3^{\circ}$ to align the femoral component with the EA. In valgus knees, it is common to externally rotate the cutting block to $5^{\circ}$ to compensate for lateral condyle hypoplasia. This has been shown to exist in valgus arthritic knees in a cadaver study [6]. However, more recent MRI studies have shown that there is considerable heterogeneity between the relationship of the EA to the PCA and that the PCA vs. EA relationship is not as deranged as previously hypothesised $[7,8]$.

The aim of the current study was to determine the impact of increasing coronal deformity, specifically valgus deformity, on the relationship between the PCA and EA utilising a large series of MRIs performed on arthritic knees.

\section{Methods}

We analysed 474 consecutive MRIs from 398 patients who had undergone total knee replacement by 2 surgeons. The purpose of the MRIs was to create patient-specific cutting blocks for the Biomet Signature patient-specific system for use during surgery.

Ankle, hip and knee MRI of the affected limb were performed according to the Biomet Signature protocol (Warsaw, NJ). Using Mimics software (Materialise,
Leuven, Belgium), the MRI sequences were converted into representative three-dimensional models of the knee. From these images, the Signature software engineers determined overall limb alignment, EA, PCA, Whiteside's line and the valgus cut angle, defined as the difference between the anatomical and mechanical axis of the femur. This data for each individual patient is available for their surgeon from the Signature Online Management System. No changes in these parameters were made by the primary surgeon.

The mechanical axis of the limb was determined on axial cuts of the hip and ankle (Fig. 1). The femoral head and ipsilateral talus were fit within a circle and a point corresponding to the centre chosen. The line connecting these points was used to determine the mechanical axis of the limb. This is a non-weight-bearing mechanical axis.

The normal mechanical axis of the limb is defined by a line passing from the centre of the femoral head to the centre of the talus. This line should pass through the centre of the knee. Valgus malalignment is defined by a mechanical axis which passes lateral to the centre of the knee while varus malalignment is defined by a mechanical axis which passes medial to the centre of the knee.

The epicondylar axis (EA) was defined by a line between the most prominent point on the lateral femoral epicondyle and the sulcus of the medial femoral epicondyle. These points correspond to the origin of the respective collateral ligaments. Whiteside's line was defined by a line connecting the deepest point of

\section{Axial View}

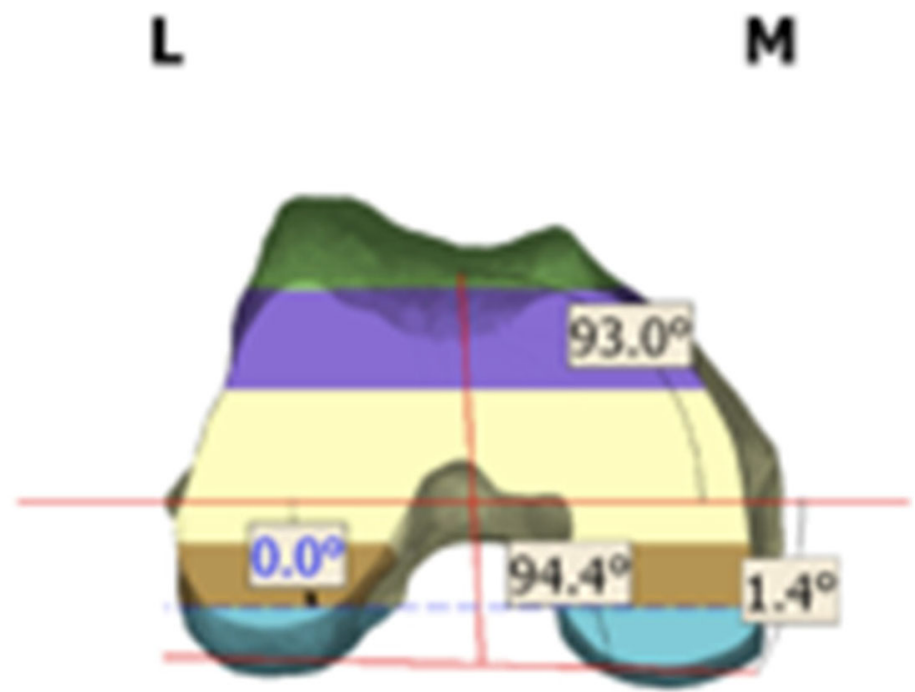

Fig. 1 Biomet Signature graphic comparing axes 
the trochlea groove and a point at the top of the femoral notch. The PCA was defined by a line tangent to the most prominent point of the posterior femoral condyles.

Age and operative side was recorded for each patient. The direction (varus or valgus) and degree of coronal deformity, the relationship between the PCA vs. EA, AP vs. EA and AP vs. PCA were obtained from the surgical planning template in the Signature Online Management System. To study the effect of increasing coronal deformity on the relationship of PCA vs. EA, we divided the valgus and varus groups into $0-5^{\circ},>5-10^{\circ}$ and $>10^{\circ}$ of deformity.

Statistical significance, defined as a $P$ value $<0.05$ was determined using Student's $t$ test, and descriptive measures including mean, median, standard deviation and confidence intervals were ascertained.

\section{Results}

There were 382 knees in 313 patients (mean age 65.7) in the varus group and 92 knees in 85 patients (mean age 67.1 ) in the valgus group. The mean non-weight-bearing coronal deformity was $6.2^{\circ}$ (95\% CI 5.92-6.57) in the varus group while the valgus group average deformity was $5.4^{\circ}$ (95\% CI 4.62-6.18) (Table 1).

In the valgus group there was statistically greater external rotation $(\mathrm{P}<0.05)$ of the EA from the PCA with a mean of $2.52^{\circ}$ (median $2.7^{\circ}, 95 \%$ CI 2.21-2.834, range $1.9^{\circ}$ to $6^{\circ}$ ) compared to the varus group with a mean of $2.03^{\circ}$ (median $1.95^{\circ}, 95 \%$ CI $1.86-2.201$, range $-3.9^{\circ}$ to $6.9^{\circ}$ ) (Table 2 \& Fig. 2).

Subgroup analysis of patients with larger coronal deformity for both varus $\left(0-5^{\circ},>5-10^{\circ}\right.$ and $\left.>10^{\circ}\right)$ and valgus $\left(0-5^{\circ},>5-10^{\circ}\right.$ and $\left.>10^{\circ}\right)$ showed no statistical difference $(P>0.05)$ in the EA vs. PCA relationship than those with lesser coronal deformity (Tables 3 and 4).

With respect to the relationship of the AP vs. PCA axes, in the valgus group, there was statistically greater external rotation of the AP relative to the PCA with a mean $95.78^{\circ}$ (95\% CI 95.25-96.31, range $87.8^{\circ}$ to $100.6^{\circ}$ ) compared to the varus group with mean $94.94^{\circ}$ (95\% CI 94.16-94.72, range $85.8^{\circ}$ to $104.8^{\circ}$ ) (Tables 2).

With respect to the AP vs. EA relationship, in the valgus group, the mean external rotation of the AP from

Table 1 Group characteristics

\begin{tabular}{lll}
\hline & Varus & Valgus \\
\hline$N$ & 382 & 92 \\
Avg. age & 65.7 & 67.1 \\
Avg. coronal alignment & 6.1 & 5.4 \\
\hline
\end{tabular}

Table 2 Relationship of distal femoral axes

\begin{tabular}{llll}
\hline & Varus & Valgus & Significance \\
\hline Mean & 2.03 & 2.52 & $P<0.05$ \\
PCA vs. EA & $(95 \%$ Cl 1.86-2.20) & $(95 \%$ Cl 2.21-2.83) & \\
Mean & 94.94 & 95.78 & $P<0.05$ \\
PCA vs. AP & $(95 \%$ Cl 94.7-95.2) & $(95 \%$ Cl 95.3-96.3) & \\
Mean & 92.93 & 93.27 & $P>0.05$ \\
EA vs. AP & $(95 \%$ Cl 92.7-93.2) & $(95 \%$ Cl 92.8-93.8) & \\
\hline
\end{tabular}

the EA was $93.27^{\circ}$ (95\% CI 92.8-93.8) and this was not statistically significant $(P>0.05)$ from the varus group mean of $92.93^{\circ}$ (95\% CI 92.7-93.2) (Table 2).

As discussed, in TKA, the aim is to align the femoral component rotation axis to the EA and the acceptable range is within $\pm 3^{\circ}$ of this axis. In varus knees (mean $2.0^{\circ}$ ER of EA vs. PCA), if an attempt to align the femoral component parallel to the EA by externally rotating the femoral cutting block $3^{\circ}$ is performed, we determined in this series that 11\% (42 of 382) of femoral components will lie outside the acceptable range. In valgus knees (mean $2.5^{\circ}$ ER of EA vs. PC), if the cutting block is externally rotated by $3^{\circ}$ or $5^{\circ}$, then $6.5 \%$ (6 of 92) and $33.7 \%$ (31 of 92) of femoral components, respectively, will lie outside of the accepted range.

\section{Discussion}

Placement of the femoral component in the arthritic knee requires an understanding of the bony changes that may occur in the distal femur. Cadaver studies have previously shown that changes in the lateral femoral condyle of the valgus knee alter the distal femoral anatomy, specifically leading to derangement of the PCA [6].

In our MRI-based study, we did not find a significant difference in the relationship between PCA vs. EA $\left(2.5^{\circ}\right.$ vs. $\left.2.0^{\circ}\right)$ and $\mathrm{PCA}$ vs. AP $\left(95.78^{\circ}\right.$ vs. $\left.94.94^{\circ}\right)$ in valgus and varus knees respectively. Our results are consistent with other MRI-based studies which have also reported that the bony changes in the valgus knee do not alter the PCA as drastically as once suggested $[7,8]$. It is however possible that lateral femoral hypoplasia, that is the posterior as well as the distal surfaces of the lateral condyle are affected, is only seen with larger valgus deformities. In our study, the mean varus deformity was $6.2^{\circ}$ and mean valgus deformity was $5.4^{\circ}$. An MRI-based study by Matsuda et al., with an average valgus deformity of $13.3 \pm 4.9^{\circ}$, demonstrated a $5^{\circ}$ difference in the rotation of the PCA vs. EA compared to varus and normal knees [9]. In our study, we did not find a significant difference in PCA vs. EA between knees 


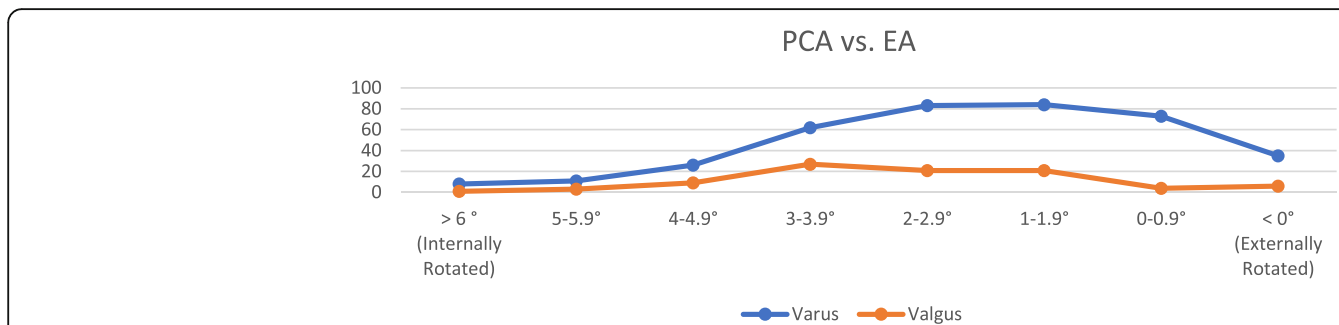

Fig. 2 Graphical distribution of PCA vs. EA

with a valgus deformity of $<5^{\circ}, 5-10^{\circ}$ and $>10^{\circ}$. However, we had only 7 knees in the $>10^{\circ}$ group.

Alignment of the femoral component in the axial plane parallel to the EA has been recommended as this is considered to be the functional axis about which knee flexion and extension occurs [10-14]. There is considerable variability, even amongst experienced surgeons, in accurately determining this axis intraoperatively [4]. The PCA, on the other hand, is much easier to identify intraoperatively, and as such the relationship of the PCA to the EA can be used to assist in alignment of the femoral component to the EA.

The relationship of PCA to EA, however, is not constant. In our study, there was considerable variation in the relationship between PCA to EA which ranged from external rotation of $2-3^{\circ}$ and internal rotation of up to $6^{\circ}$ in both varus and valgus knees. Many conventional jig posterior referenced TKA systems recommend external rotation of the femoral cutting jig to $3^{\circ}$ in varus knees and $5^{\circ}$ in valgus knees relative to the native PCA in order to align the femoral component with the EA. Based on our results, choosing to externally rotate the femoral cutting guide by $3^{\circ}$ relative to the native PCA would lead to a rotational malalignment $\left(>3^{\circ}\right.$ from EA) in femoral component placement in $11 \%$ of varus knees and $6.5 \%$ of valgus knees. Externally rotating the femoral cutting guide by $5^{\circ}$ from the PCA in valgus knees would lead to a rotational malalignment in 33.7\%. A 2010 study using MRI to assess femoral component rotation reported the number of $>3^{\circ}$ outliers in conventional jig TKA systems was $22.9 \%$ [15].

Malrotation of the femoral component in the axial plane leads to patellofemoral mal-tracking, overtigh

Table 3 Varus subgroup analysis

\begin{tabular}{llll}
\hline & $0-5^{\circ}$ & $>5-10^{\circ}$ & $>10^{\circ}$ \\
\hline$N$ & 166 & 176 & 40 \\
Mean & 2.16 & 2.02 & 1.53 \\
Cl & $1.91-2.41$ & $1.76-2.28$ & $0.98-2.08$ \\
SD & 1.642 & 1.735 & 1.792 \\
\hline
\end{tabular}

tening of the lateral (excessive ER) or medial (excessive IR) compartments, especially in flexion and thus an overall kinematic mismatch. [4, 9, 16-18] Additionally, femoral component malalignment in the axial plane has been associated with poor patient-reported outcomes, accelerated wear and an increased revision rate $[5,11,19]$.

There were limitations to this study. The overall limb alignment was calculated using a non-weight-bearing imaging modality. Use of weight-bearing $\mathrm{x}$-rays would probably accentuate the deformity in many cases. This may affect the validity of our results. Furthermore, it is possible that the posterior aspect of the lateral femoral condyle is only significantly affected with larger valgus deformities. We may not have observed these changes due to the small number of patients in this study with large valgus deformities $\left(>10^{\circ}\right)$. Finally, determination of the various axes was performed by an unknown number of software engineers based on single points and subject to error. We do not have knowledge of the inter- and intra-observer reliability of these measurements.

\section{Conclusion}

Our study showed that valgus deformity does not significantly alter the relationship of the PCA vs. the EA and AP axis as previous studies suggest. The relationship of the PCA to the EA and AP axes in both varus and valgus deformity is quite heterogeneous and not reliable in isolation for proper femoral component rotational alignment. External rotation beyond $3^{\circ}$ from PCA in valgus knees may lead to significant femoral component malrotation in a large proportion cases.

Table 4 Valgus subgroup analysis

\begin{tabular}{llll}
\hline & $0-5^{\circ}$ & $>5-10^{\circ}$ & $>10^{\circ}$ \\
\hline$N$ & 54 & 31 & 7 \\
Mean & 2.48 & 2.42 & 3.34 \\
Cl & $2.06-2.90$ & $1.90-2.96$ & $2.88-3.81$ \\
SD & 1.581 & 1.494 & 0.624 \\
\hline
\end{tabular}




\section{Abbreviations}

AP: Anteroposterior axis; EA: Epicondylar axis; MRI: Magnetic resonance imaging; PCA: Posterior condylar; TKA: Total knee arthroplasty

\section{Acknowledgements}

No other acknowledgements applicable

\section{Declarations of interest}

None

\section{Funding}

No Funding was received for this project.

\section{Availability of data and materials}

All data was obtained from the Signature Online Management System for two of the corresponding authors (AL and AG). This is password encrypted data available to the operating consultant surgeon. De-identified data was recorded and statistical analysis of said data is being held in a password protected computer and available on request. Please contact author for data requests

\section{Authors' Contribution}

DC contributed to the study design, data collection, data analysis and statistical interpretation and is the primary author of paper.AG contributed to the study design, use of patient's data from Biomet Signature PSI system and proofreading.AL contributed to the conception, study design, gained ethics approval, use of patient's data from Biomet Signature PSI system and proofreading. All authors read and approved the final manuscript.

\section{Authors' information}

DC - Advanced Trainee in Orthopaedic Surgery, Year 4 Southside region (AOA), Bachelor of Science (Anatomy) and Masters of Science (Physiology) (McGill University, Montreal, Quebec, Canada) MBBS (University of Sydney, Sydney, NSW, Australia)

AG - MBBS (University of NSW, Kensington, NSW, Australia), Fellow of Royal Australian College of Surgeons (Orthopaedics), Visiting Medical Officer (Sydney Adventist Hospital, Macquarie Hospital, Norwest Private Hospital) AL - MBBS (University of Sydney, Sydney, NSW, Australia), PhD ( University of Sydney, Sydney, NSW, Australia) Fellow of Royal Australian College of Surgeons (Orthopaedics), Senior Lecturer University of Sydney, Visiting Medical Officer (Sydney Adventist Hospital, Macquarie Hospital, Norwest Private Hospital),

\section{Ethics approval and consent to participate}

Ethics for this paper was given by the University of New South Wales, UNSW Ref \# HC12169. A waiver for individual patient consent was granted by the approving committee.

Patients consented for imaging and creation of patient specific instrumentation with the individual surgeons however there was no direct communication between the company and patients.De-identified data was recorded retrospectively and no individual data was published in this manuscript. No consent was needed to participate in this study. Zimmer Biomet provided written consent for publication of an example of the 'Signature' surgical template image. This is available on request.

\section{Consent for publication}

Not applicable

\section{Competing interests}

The authors declare that they have no competing interests.

\section{Publisher's Note}

Springer Nature remains neutral with regard to jurisdictional claims in published maps and institutional affiliations.
Received: 25 July 2018 Accepted: 15 March 2019

Published online: 02 April 2019

\section{References}

1. Berger RA, Rubash HE, Seel MJ, Thompson WH, Crossett LS. Determining the rotational alignment of the femoral component in total knee arthroplasty using the epicondylar axis. Clin Orthop Relat Res. 1993;286:40-7.

2. Griffin FM, Insall JN, Scuderi GR. The posterior condylar angle in osteoarthritic knees. J Arthroplast. 1998:13:812-5.

3. Yoshioka Y, Siu D, Cooke TD. The anatomy and functional axes of the femur. J Bone Jt Surg Am. 1987:69:873-80.

4. Kinzel V, Ledger M, Shakespeare D. Can the epicondylar axis be defined accurately in total knee arthroplasty? Knee. 2005;12:293-6.

5. Victor J. Rotational alignment of the distal femur: a literature review. Orthop Traumatol Surg Res. 2009:95:365-72.

6. Arima J, Whiteside LA, MCCarthy DS, White SE. Femoral rotational alignment, based on the antero-posterior axis, in total knee arthroplasty in a valgus knee. A technical note. J Bone Jt Surg Am. 1995;77:1331-4.

7. Paternostre F, Schwab P-E, Thienpont E. The combined Whiteside's and posterior condylar line as a reliable reference to describe axial distal femoral anatomy in patient-specific instrument planning. Knee Surg Sports Traumatol Arthrosc. 2014:22:3054-9.

8. Amaranth JE, Moonapar TR, Sorial RM. Defining distal femoral anatomy for rotational alignment in total knee arthroplasty: a magnetic resonance imaging-based study. ANZ J of Surgery. 2014;84(11):852-5.

9. Matsuda S, Miura H, Nagamine R, Mawatari T, Tokunaga M, et al. Anatomical analysis of the femoral condyle in normal and osteoarthritic knees. J Orthop Res. 2004;22(1):104-9.

10. Miller MC, Berger RA, Petrella AJ, Karmas A, Rubash HE. Optimizing femoral component rotation in total knee arthroplasty. Clin Orthop Relat Res. 2001; 392:38-45.

11. Newbern DG, Faris PM, Ritter MA, Keating EM, Meding JB, Berend ME. A clinical comparison of patellar tracking using the transepicondylar axis and the posterior condylar axis. J Arthroplast. 2006;21:1141-6.

12. Asano T, Akagi M, Nakamura T. The functional flexion-extension axis of the knee corresponds to the surgical epicondylar axis. J Arthroplast. 2005;20:1060-7.

13. Churchill DL, Incavo SJ, Johnson CC, Beynnon BD. The transepicondylar axis approximates the optimal flexion axis of the knee. Clin Orthop Relat Res. 1998;356:111-8

14. Hollister AM, Jatana A, Singh AK, Sullivan WW, Lupichuk AG. The axes of rotation of the knee. Clin Orthop Relat Res. 1993;290:259-68.

15. Heyse T, Tibesku C. Improved femoral component rotation in TKA using patient-specific instrumentation. Knee. 2010;21(Issue 1):268-71.

16. Akagi M, Matsusue Y, Mata T, Asada Y, Horiguchi M, lida H, Nakamura T. Effect of rotational alignment on patellar tracking in total knee arthroplasty. Clin Orthop Relat Res. 1999;366:155-63.

17. Anouchi YS, Whiteside LA, Kaiser AD, Milliano MT. The effects of axial rotational alignment of the femoral component on knee stability and patellar tracking in total knee arthroplasty demonstrated on autopsy specimens. Clin Orthop Relat Res. 1993;287:170-7.

18. Merican AM, Ghosh KM, Iranpour F, Deehan DJ, Amis AA. The effect of femoral component rotation on the kine- matics of the tibiofemoral and patellofemoral joints after total knee arthroplasty. Knee Surg Sports Traumatol Arthrosc. 2011:19:1479-87.

19. Berger RA, Crossett LS, Jacobs JJ, Rubash HE. Malrotation causing patellofemoral complications after total knee arthroplasty. Clin Orthop Relat Res. 1998;356:144-53.

Ready to submit your research? Choose BMC and benefit from:

- fast, convenient online submission

- thorough peer review by experienced researchers in your field

- rapid publication on acceptance

- support for research data, including large and complex data types

- gold Open Access which fosters wider collaboration and increased citations

- maximum visibility for your research: over $100 \mathrm{M}$ website views per year

At $B M C$, research is always in progress.

Learn more biomedcentral.com/submissions 